\title{
Gluon Transport Equation with Effective Mass and Dynamical Onset of Bose-Einstein Condensation
}

\author{
Jean-Paul Blaizot ${ }^{\mathrm{a}}$, Yin Jiang ${ }^{\mathrm{b}}$, Jinfeng Liao ${ }^{\mathrm{b}, \mathrm{c}}$ \\ ${ }^{a}$ Institut de Physique Théorique (IPhT), CNRS/URA2306, CEA Saclay, \\ F-91191 Gif-sur-Yvette, France \\ ${ }^{b}$ Physics Department and Center for Exploration of Energy and Matter, Indiana University, \\ $2401 N$ Milo B. Sampson Lane, Bloomington, IN 47408, USA \\ ${ }^{c}$ RIKEN BNL Research Center, Bldg. 510A, Brookhaven National Laboratory, Upton, NY \\ 11973, USA
}

\begin{abstract}
We study the transport equation describing a dense system of gluons, in the small scattering angle approximation, taking into account medium-generated effective masses of the gluons. We focus on the case of overpopulated systems that are driven to Bose-Einstein condensation on their way to thermalization. The presence of a mass modifies the dispersion relation of the gluon, as compared to the massless case, but it is shown that this does not change qualitatively the scaling behavior in the vicinity of the onset.
\end{abstract}

\section{Introduction}

In previous papers $[1,2]$ it was argued that a dense system of gluons such as those created in the early stages of an ultra-relativistic heavy ion collision, could be driven to Bose-Einstein condensation, as the system evolves towards thermal equilibrium. This was inferred from a detailed study of the kinetic equation that takes into account 2 to 2 scattering, in the small scattering angle approximation. Overpopulation means that the dimensionless number $n / \epsilon^{3 / 4}$, where $n$ is the number density and $\epsilon$ the energy density, exceeds its value in equilibrium. An overpopulated system has too many gluons, relative to its total energy, to be accommodated in a Bose-Einstein distribution, and thermal equilibrium requires the formation of a condensate.

Of course, such a condensate will develop provided the approach to thermal equilibrium proceeds with conservation of both energy and particle number. While energy is certainly conserved, inelastic processes of various kinds may change the number of gluons (see e.g. $[3,4])^{1}$, eventually preventing the formation of a condensate in the true equilibrium state. However, particle number may be approximately conserved during much of the evolution, and this could

\footnotetext{
${ }^{1}$ Note that quark production, although it decreases the number of gluons, does not necessarily hinds the formation of a condensate[4].
}

Preprint submitted to Elsevier

July 13, 2015

(C) 2015. This manuscript version is made available under the Elsevier user license http://www.elsevier.com/open-access/userlicense/1.0/ 
be enough to approach condensation. Indeed, transport calculations indicate that the amplification of soft modes is a very rapid process, that a chemical potential is indeed dynamically generated and that the onset for condensation can be reached on short time scales. This is confirmed by calculations using the small angle approximation [2], as well as complete solution of the Boltzmann equation $[5,7]$. There are also indications that inelastic processes could accelerate the amplification of soft modes [6], while the authors of Refs. [8] and [9] seemingly reach a different conclusion.

Clearly the analysis of inelastic scatterings requires further work, but this is beyond the scope of this paper. Motivation for studying the possibility for gluons to condense comes of course from the desire to better understand how matter produced in a high energy nucleus-nucleus collision evolves towards local thermal equilibrium (see $[10,11]$ for a recent review). But, as we already emphasized in [2], the general issue of the dynamical formation of a condensate is an interesting problem in itself. It is of relevance in the context of cosmology (see e.g. [12]), or cold atom physics (see e.g. [13]). It has be studied using kinetic theory, or classical field simulations (see e.g. $[14,15]$ ). In the context of Quantum Chromodynamics, the nature of the condensate remains an interesting, but unsolved, question (for a recent study in a related context, see [16]).

Our goal in this paper is to pursue our general study of the phenomenon within kinetic theory, using a transport equation that incorporates properly the effects of Bose statistics. The fact that the interactions are long range interactions validate the use of the small angle approximation which reduces the transport equation to a Fokker-Planck equation, much easier to solve than the Boltzmann equation, thereby providing more direct analytical insight. This paper, as well as a companion paper, addresses issues that were not discussed in Ref. [2] which is limited to the study of the onset of condensation. We want to extend our work so as to be able to obtain a complete dynamical description of the approach to equilibrium including the formation of a condensate. In order to do so, we need to attribute finite masses to the gluons. Such masses are automatically generated by the coupling to thermal fluctuations, and the proper transport equations that incorporate such self-energy corrections could be derived from first principles. However, for the purpose of the present study, it is sufficient to just give the gluons a mass, and correct appropriately the scattering matrix element. In fact two masses will be introduced. The screening mass $m_{D}$ regulates the infrared behavior of the collision kernel. The other mass, $m$, modifies the dispersion relation, and one of the issues that we want to study is how this modification changes the onset of Bose-Einstein condensation. We shall see that, in fact, qualitatively it does not. Finally, the role of the mass $m$ is to allow a clear definition of the equations that describe the evolution of the system beyond the onset, that is, in the presence of the condensate. This will be discussed in a companion paper [17].

The outline of this paper is as follows. In the next section, we re-derive the approximate form of the transport equation in the small scattering angle approximation, paying attention to the presence of finite masses, and emphasizing the differences with the massless case discussed in [2]. In section 3, we 
present results of numerical solutions of the transport equation, illustrating the role of the mass on generic features of thermalization in both the underpopulated and overpopulated situations. In section 4, we focus on the critical regime that accompanies the onset of condensation. We show in particular that the change of the dispersion relation, from ultra-relativistic in the massless case, to non-relativistic in the massive case, does not change qualitatively the scaling regime. Details on the analytic calculation, as well as on the numerical solution, are given in two appendices.

\section{Derivation of the Transport Equation in the Massive Case}

In this Section, we derive the transport equation, under the approximation of small scattering angle, taking into account medium-induced effective masses for both the colliding gluons and the exchange gluons.

\subsection{Generalities}

As in our previous papers [1,2], we assume a transport equation for the single particle distribution of the following form

$$
\begin{aligned}
\mathcal{D}_{t} f_{1} & =C[f] \\
& =\frac{1}{2} \int \frac{d^{3} \boldsymbol{p}_{2}}{(2 \pi)^{3} 2 E_{2}} \frac{d^{3} \boldsymbol{p}_{3}}{(2 \pi)^{3} 2 E_{3}} \frac{d^{3} \boldsymbol{p}_{4}}{(2 \pi)^{3} 2 E_{4}} \frac{1}{2 E_{1}}\left|\mathcal{M}_{12 \rightarrow 34}\right|^{2} \\
& \times(2 \pi)^{4} \delta\left(P_{1}+P_{2}-P_{3}-P_{4}\right)\left\{f_{3} f_{4}\left(1+f_{1}\right)\left(1+f_{2}\right)-f_{1} f_{2}\left(1+f_{3}\right)\left(1+f_{4}\right)\right\}
\end{aligned}
$$

where

$$
\mathcal{D}_{t} \equiv \partial_{t}+\boldsymbol{v}_{1} \cdot \nabla
$$

and the factor $1 / 2$ in front of the integral is a symmetry factor. Summation over color and polarization is performed on the gluons 2,3,4; average over color and polarization is performed for gluon 1. The distribution function $f$ is a scalar object (i.e., independent of color and spin):

$$
f(\boldsymbol{x}, \boldsymbol{p})=\frac{(2 \pi)^{3}}{2\left(N_{c}^{2}-1\right)} \frac{d N}{d^{3} \boldsymbol{x} d^{3} \boldsymbol{p}},
$$

where $N$ denotes the total number of gluons. In other words, $f$ denotes the number of gluons of a given spin and color in the phase-space element $d^{3} \boldsymbol{x} d^{3} \boldsymbol{p} /(2 \pi)^{3}$. We consider a uniform system, so that $f$ is in fact independent of $\boldsymbol{x}$. Also, in this paper, we consider a non-expanding system, so that $\mathcal{D}_{t}=\partial_{t}$. Finally, $P_{i}$ denotes the four-momentum of particle $i, P_{i}=\left(E_{\boldsymbol{p}_{i}}, \boldsymbol{p}_{i}\right)$.

In this paper, contrary to our previous work, we assume that the gluons carry a small mass, arising from their interactions with the medium. This is a crude approximation to the true self-energy, but our main goal here is not to give a quantitative description of the phenomenon, but rather to study how 
the onset of Bose-Einstein condensation is affected by such a mass. We believe that a more complete, but much more difficult, treatment would not change our main conclusions. The major change brought by the presence of the mass is that of the dispersion relation of the modes. Thus the energy of a particle with momentum $\boldsymbol{p}$ is $E_{\boldsymbol{p}}=\sqrt{\boldsymbol{p}^{2}+m^{2}}$.

It is convenient to express the final momenta in terms of the initial ones and of the momentum $\boldsymbol{q}$ transferred in the collision. We set

$$
\boldsymbol{p}_{3}=\boldsymbol{p}_{1}+\boldsymbol{q}, \quad \boldsymbol{p}_{4}=\boldsymbol{p}_{2}-\boldsymbol{q} .
$$

One can then perform the integrations over $\boldsymbol{p}_{3}$ and $\boldsymbol{p}_{4}$ using the three-momentum delta function. We get

$$
C[f]=\int_{\boldsymbol{p}_{2}} \int_{\boldsymbol{q}} w\left(\boldsymbol{p}_{1}, \boldsymbol{p}_{2}, \boldsymbol{q}\right)\left\{f_{12 \rightarrow 34}\right\},
$$

with

$$
w\left(\boldsymbol{p}_{1}, \boldsymbol{p}_{2}, \boldsymbol{q}\right) \equiv \frac{\pi \delta\left(E_{1}+E_{2}-E_{3}-E_{4}\right)}{16 E_{1} E_{2} E_{3} E_{4}}\left|\mathcal{M}_{12 \rightarrow 34}\right|^{2},
$$

and

$$
\left\{f_{12 \rightarrow 34}\right\} \equiv f_{3} f_{4}\left(1+f_{1}\right)\left(1+f_{2}\right)-f_{1} f_{2}\left(1+f_{3}\right)\left(1+f_{4}\right),
$$

where $f_{i}$ is a shorthand notation for $f_{\boldsymbol{p}_{i}}$. The quantity $w\left(\boldsymbol{p}_{1}, \boldsymbol{p}_{2}, \boldsymbol{q}\right)$ may be interpreted as the rate of collisions of particle 1 with particle 2 , in which momentum $\boldsymbol{q}$ is transferred to particle 1 , its momentum $\boldsymbol{p}_{1}$ becoming $\boldsymbol{p}_{1}+\boldsymbol{q}$. Note that the symmetry factor is included in the definition of $w$. The (dimensionless) matrix element $\mathcal{M}_{12 \rightarrow 34}$ will be discussed shortly. It is understood in the expressions above that the momenta $\boldsymbol{p}_{3}$ and $\boldsymbol{p}_{4}$ are expressed in terms of $\boldsymbol{p}_{1}, \boldsymbol{p}_{2}, \boldsymbol{q}$ according to (4). Also, we used the shorthand for momentum integration

$$
\int_{\boldsymbol{p}} \equiv \int \frac{\mathrm{d}^{3} \boldsymbol{p}}{(2 \pi)^{3}}
$$

Note that the symmetries of the matrix element (see below) entail the property

$$
w\left(\boldsymbol{p}_{1}, \boldsymbol{p}_{2},-\boldsymbol{q}\right)=w\left(\boldsymbol{p}_{1}, \boldsymbol{p}_{2}, \boldsymbol{q}\right)=w\left(\boldsymbol{p}_{2}, \boldsymbol{p}_{1},-\boldsymbol{q}\right) .
$$

\subsection{The small scattering angle approximation and the Fokker-Planck equation}

Under the small scattering angle approximation, the momenta of incident particles get changed very little during each collision, and the kinetic equation can be approximated by a Fokker-Planck equation in momentum space [18]. Following a standard procedure [19], we write the collision integral as

$$
C[f]=-\nabla \cdot \mathcal{J}=-\frac{\partial \mathcal{J}_{i}}{\partial p_{i}} .
$$


Note the gradient $\boldsymbol{\nabla}$ in the above, and for the rest of this paper, is the momentum space gradient i.e. $\boldsymbol{\nabla}=\boldsymbol{\nabla}_{\boldsymbol{p}}$. In order to estimate $\mathcal{J}_{i}$, the component of the current (of particles 1 ) in the direction $i$ (with $i=1,2,3$ ), we count the number of particles that, as a result of collisions during the interval $\mathrm{d} t$, cross a surface element orthogonal to the direction $i$ and located at a particular value $\overline{\boldsymbol{p}}$ of $\boldsymbol{p}_{1}$. An elementary analysis yields

$$
\begin{aligned}
\mathcal{J}_{i} & =\int_{\boldsymbol{q}, q_{i}>0} \int_{\boldsymbol{p}_{2}} \int_{\bar{p}_{i}-q_{i}}^{\bar{p}_{i}} d p_{i} w\left(\boldsymbol{p}_{1}, \boldsymbol{p}_{2}, \boldsymbol{q}\right) \\
& \times\left\{f_{\boldsymbol{p}_{1}} f_{\boldsymbol{p}_{2}}\left(1+f_{\boldsymbol{p}_{1}+\boldsymbol{q}}\right)\left(1+f_{\boldsymbol{p}_{2}-\boldsymbol{q}}\right)-\left(1+f_{\boldsymbol{p}_{1}}\right)\left(1+f_{\boldsymbol{p}_{2}}\right) f_{\boldsymbol{p}_{1}+\boldsymbol{q}} f_{\boldsymbol{p}_{2}-\boldsymbol{q}}\right\}
\end{aligned}
$$

where the $\boldsymbol{q}$-integration is restricted to positive components $q_{i}$. In the small angle approximation the combination of statistical factors simplifies into

$$
\begin{aligned}
& f_{\boldsymbol{p}_{1}} f_{\boldsymbol{p}_{2}}\left(1+f_{\boldsymbol{p}_{1}+\boldsymbol{q}}\right)\left(1+f_{\boldsymbol{p}_{2}-\boldsymbol{q}}\right)-\left(1+f_{\boldsymbol{p}_{1}}\right)\left(1+f_{\boldsymbol{p}_{2}}\right) f_{\boldsymbol{p}_{1}+\boldsymbol{q}} f_{\boldsymbol{p}_{2}-\boldsymbol{q}} \\
& \approx \boldsymbol{q} \cdot\left[h_{\boldsymbol{p}_{1}}(\boldsymbol{\nabla} f)_{\boldsymbol{p}_{2}}-h_{\boldsymbol{p}_{2}}(\boldsymbol{\nabla} f)_{\boldsymbol{p}_{1}}\right]+\mathcal{O}\left(\boldsymbol{q}^{2}\right),
\end{aligned}
$$

where we have introduced the notation $h_{\boldsymbol{p}} \equiv f_{\boldsymbol{p}}\left(1+f_{\boldsymbol{p}}\right)$. In addition, we notice that, in this approximation, the energy conservation implies

$$
0=\boldsymbol{q} \cdot \boldsymbol{v}_{1}-\boldsymbol{q} \cdot \boldsymbol{v}_{2}+\mathcal{O}\left(\boldsymbol{q}^{2}\right) .
$$

where $\boldsymbol{v}_{i} \equiv \frac{p_{i}}{E_{i}}$. By taking all these together we obtain the following leading order expression for the momentum flux:

$$
\mathcal{J}^{i}=\frac{1}{2} \int_{\boldsymbol{q}} \int_{\boldsymbol{p}_{2}} \boldsymbol{q}^{i} w\left(\boldsymbol{p}_{1}, \boldsymbol{p}_{2}, \boldsymbol{q}\right) \boldsymbol{q} \cdot\left[h_{\boldsymbol{p}_{1}}(\boldsymbol{\nabla} f)_{\boldsymbol{p}_{2}}-h_{\boldsymbol{p}_{2}}(\boldsymbol{\nabla} f)_{\boldsymbol{p}_{1}}\right] .
$$

Note that in the above equation we have relaxed the constraint $q_{i}>0$ on the $\boldsymbol{q}$-integration, dividing the result by 2 (using the fact that the integrand is even in $\boldsymbol{q}$, see Eqs. (9)). We can rewrite the current as follows

$$
\mathcal{J}^{i}=\int_{\boldsymbol{p}_{2}} B^{i j}\left(\boldsymbol{p}_{1}, \boldsymbol{p}_{2}\right)\left[h_{\boldsymbol{p}_{1}}\left(\boldsymbol{\nabla}^{j} f\right)_{\boldsymbol{p}_{2}}-h_{\boldsymbol{p}_{2}}\left(\boldsymbol{\nabla}^{j} f\right)_{\boldsymbol{p}_{1}}\right],
$$

with the (dimensionless) angular tensor

$$
B^{i j}\left(\boldsymbol{p}_{1}, \boldsymbol{p}_{2}\right)=\frac{1}{2} \int_{\boldsymbol{q}} \boldsymbol{q}^{i} \boldsymbol{q}^{j} w\left(\boldsymbol{p}_{1}, \boldsymbol{p}_{2}, \boldsymbol{q}\right) .
$$

\subsection{Conservation laws}

The particle number conservation is obvious due to the structure of the collision term as the divergence of a current:

$$
\int_{\boldsymbol{p}_{1}} C\left[f_{\boldsymbol{p}_{1}}\right]=0
$$


To prove the energy conservation requires a little more effort

$$
\begin{aligned}
\int_{\boldsymbol{p}_{1}} E_{\boldsymbol{p}_{1}} C\left[f_{\boldsymbol{p}_{1}}\right] & =-\int_{\boldsymbol{p}_{1}} E_{\boldsymbol{p}_{1}} \boldsymbol{\nabla}_{\boldsymbol{p}_{1}} \cdot \mathcal{J}\left(\boldsymbol{p}_{1}\right) \\
& =-\int_{\boldsymbol{p}_{1}} \boldsymbol{\nabla}_{\boldsymbol{p}_{1}} \cdot\left[E_{\boldsymbol{p}_{1}} \mathcal{J}\left(\boldsymbol{p}_{1}\right)\right]+\int_{\boldsymbol{p}_{1}}\left(\boldsymbol{\nabla}_{\boldsymbol{p}_{1}} E_{\boldsymbol{p}_{1}}\right) \cdot \mathcal{J}\left(\boldsymbol{p}_{1}\right) \\
& =\int \frac{d^{3} \boldsymbol{p}_{1}}{(2 \pi)^{3}} \boldsymbol{v}_{1} \cdot \mathcal{J}\left(\boldsymbol{p}_{1}\right) \\
& =\frac{1}{2} \int_{\boldsymbol{p}_{1}} \int_{\boldsymbol{p}_{2}} \int_{\boldsymbol{q}} w\left(\boldsymbol{p}_{1}, \boldsymbol{p}_{2} ; \boldsymbol{q}\right) \\
& =\frac{1}{2} \int_{\boldsymbol{p}_{1}} \int_{\boldsymbol{p}_{2}} \int_{\boldsymbol{q}} w\left(\boldsymbol{p}_{1}, \boldsymbol{p}_{2} ; \boldsymbol{q}\right) \\
& \quad \times\left[\left(\boldsymbol{v}_{1} \cdot \boldsymbol{q}\right) h_{\boldsymbol{p}_{1}}(\boldsymbol{q} \cdot \boldsymbol{\nabla} f)_{\boldsymbol{p}_{2}}-\left(\boldsymbol{v}_{2} \cdot \boldsymbol{q}\right) h_{\boldsymbol{p}_{2}}(\boldsymbol{q} \cdot \boldsymbol{\nabla} f)_{\boldsymbol{p}_{1}}\right] \\
& =0 \quad
\end{aligned}
$$

where in the last steps we have used Eq.(13) and Eqs. (9).

\subsection{The matrix element}

The matrix element for (in vacuum) gluon-gluon scattering $(1+2 \rightarrow 3+4)$ reads (spin and color averaged for 1 , and summed for 2,3,4)

$$
|\mathcal{M}|^{2}=128 \pi^{2} \alpha_{s}^{2} N_{c}^{2}\left[3-\frac{t u}{s^{2}}-\frac{s u}{t^{2}}-\frac{t s}{u^{2}}\right], \quad 128 \pi^{2} \alpha_{s}^{2} N_{c}^{2}=72 g^{4},
$$

with $s, t, u$ the standard Mandelstam variables:

$$
s=\left(P_{1}+P_{2}\right)^{2}, \quad t=\left(P_{1}-P_{3}\right)^{2}, \quad u=\left(P_{1}-P_{4}\right)^{2} .
$$

In the small scattering angle approximation, the dominant contributions come from the kinematic regions $t \approx 0$ and $u \approx 0$. With the vacuum matrix element and in massless case, one would have the following approximation:

$$
|\mathcal{M}|^{2}=72 g^{4}\left[3-\frac{t u}{s^{2}}-\frac{s u}{t^{2}}-\frac{t s}{u^{2}}\right] \approx 72 g^{4}\left[-\frac{s u}{t^{2}}-\frac{t s}{u^{2}}\right] \approx 144 g^{4} \frac{s^{2}}{t^{2}},
$$

where in the last step we have used the fact that the two contributions are equal, and, for massless particles, $u=-(s+t) \approx-s$ (for $t \approx 0)$.

Now we consider the modifications of the matrix elements that need to be taken into account when the gluons are massive. There are two distinct physical effects. One is the screening of the $t$-channel singularity. This should be taken care of by separating the transverse and the longitudinal channels, and including the proper polarization tensors in the exchange gluons. In this paper we simply modify the denominator by substituting $t \rightarrow t-m_{D}^{2}$, with $m_{D}$ a screening mass whose main role here is that of a regulator. The other physical effect is coming 
from self-energy corrections on the external lines. As already mentioned, we simply take these into account by giving the gluon a small thermal mass $m$, allowing $m$ to differ from $m_{D}$. In summary, we replace the matrix element derived for massless particles by

$$
|\mathcal{M}|^{2} \rightarrow 144 g^{4} \frac{s^{2}}{\left(t-m_{D}^{2}\right)^{2}}, \quad s \approx 2 E_{1} E_{2}\left(1-\boldsymbol{v}_{1} \cdot \boldsymbol{v}_{2}\right),
$$

where $\boldsymbol{v}_{i}=\boldsymbol{p}_{i} / E_{i}$ is the velocity of particle $i$, and since we are interested in the small $m$ limit, we dropped a term $\sim m^{2}$ in the expression of $s$. This approximation requires $m^{2} \ll s$, which is true for the typical scattering processes involving $s$ on the order of $Q_{s}^{2}$. It shall be emphasized that even in the regime of very soft momentum, the dominant collisions that drive the evolution come from collisions with a typical momentum scale particle and in such collisions the condition $m^{2} \ll s \sim Q_{s}^{2}$ is still satisfied. With that, we then obtain, in the leading order of the small scattering angle approximation

$$
w=36 \pi g^{4} \delta\left(\boldsymbol{q} \cdot \boldsymbol{v}_{1}-\boldsymbol{q} \cdot \boldsymbol{v}_{2}\right) \frac{\left(1-\boldsymbol{v}_{1} \cdot \boldsymbol{v}_{2}\right)^{2}}{\left(\omega^{2}-\boldsymbol{q}^{2}-m_{D}^{2}\right)^{2}}
$$

where $\omega=\boldsymbol{v}_{1} \cdot \boldsymbol{q}$.

With this matrix element, the angular tensor takes the form

$$
B^{i j}\left(\boldsymbol{p}_{1}, \boldsymbol{p}_{2}\right)=18 \pi g^{4} \int_{\boldsymbol{q}} \delta\left(\boldsymbol{v}_{1} \cdot \boldsymbol{q}-\boldsymbol{v}_{2} \cdot \boldsymbol{q}\right) \boldsymbol{q}^{i} \boldsymbol{q}^{j} \frac{\left(1-\boldsymbol{v}_{1} \cdot \boldsymbol{v}_{2}\right)^{2}}{\left(\omega^{2}-\boldsymbol{q}^{2}-m_{D}^{2}\right)^{2}} .
$$

\subsection{The isotropic case}

We assume that the distribution function is a function of the energy, $f_{\boldsymbol{p}}=$ $f\left(E_{\boldsymbol{p}}\right)$ with $E_{\boldsymbol{p}}=\sqrt{\boldsymbol{p}^{2}+m^{2}}$. In such case we have the following relation

$$
\nabla f_{\boldsymbol{p}}=\boldsymbol{v} f^{\prime}\left(E_{\boldsymbol{p}}\right), \quad f^{\prime}(E) \equiv \frac{\mathrm{d} f(E)}{\mathrm{d} E},
$$

with the velocity given by $\boldsymbol{v}=\boldsymbol{p} / E_{\boldsymbol{p}}=\nabla_{p} E_{\boldsymbol{p}}$. Then we have

$$
h_{\boldsymbol{p}_{1}}\left(\boldsymbol{\nabla}^{j} f\right)_{\boldsymbol{p}_{2}}-h_{\boldsymbol{p}_{2}}\left(\boldsymbol{\nabla}^{j} f\right)_{\boldsymbol{p}_{1}}=\boldsymbol{v}_{2}^{j} h_{1} f_{2}^{\prime}-\boldsymbol{v}_{1}^{j} h_{2} f_{1}^{\prime},
$$

where we introduced the simplified notation $f_{i}=f\left(E_{i}\right)=f_{\boldsymbol{p}_{i}}$ (and similarly for $h_{i}$ ) that will be used throughout the paper.

In this isotropic case, the calculation of the angular tensor (24) simplifies. In particular, the current $\overrightarrow{\mathcal{J}}(\boldsymbol{p})$ is a vector aligned with the direction of $\boldsymbol{p}$, that is (with $p_{1}=\left|\boldsymbol{p}_{1}\right|$ and $\hat{\boldsymbol{p}}_{1}=\boldsymbol{p}_{1} / p_{1}$ )

$$
\overrightarrow{\mathcal{J}}\left(\boldsymbol{p}_{1}\right)=\hat{\boldsymbol{p}}_{1} \mathcal{J}\left(p_{1}\right) .
$$

It follows that the kinetic equation can be written as

$$
\mathcal{D}_{t} f_{1}=-\frac{1}{p_{1}^{2}} \partial_{p_{1}}\left\{p_{1}^{2} \mathcal{J}\left(p_{1}\right)\right\}
$$


The calculation presented in Appendix A yields

$$
\mathcal{J}\left(p_{1}\right)=36 \pi \alpha_{s}^{2} \int_{\boldsymbol{p}_{2}}\left(h_{1} f_{2}^{\prime}-h_{2} f_{1}^{\prime}\right) \mathcal{Z}\left(v_{1}, v_{2}\right),
$$

where the explicit expressions of the dimensionless function $\mathcal{Z}\left(v_{1}, v_{2}\right)$ is given in Appendix A.

\subsection{The massless limit}

It is shown in Appendix A.4 that the current at small momentum has the following structure

$$
-\mathcal{J}\left(p_{1} \rightarrow 0\right) \simeq 36 \pi \alpha_{s}^{2} \mathcal{L}\left[I_{a}\left(p_{1}\right) \partial_{p_{1}} f_{1}+\frac{p_{1}}{E_{1}} I_{b}\left(p_{1}\right) h_{1}\right]
$$

where $\mathcal{L}$ is a positive constant, and (see Eq. (A.25))

$$
I_{a}\left(p_{1}\right) \equiv \frac{p_{1}}{m} \int_{\boldsymbol{p}_{2}} \frac{h_{2}}{v_{2}}, \quad I_{b}\left(p_{1}\right) \equiv-\frac{p_{1}}{m} \int_{\boldsymbol{p}_{2}} \frac{f_{2}^{\prime}}{v_{2}}=-\frac{p_{1}}{m} \int_{\boldsymbol{p}_{2}} \frac{1}{v_{2}^{2}} \partial_{p_{2}} f_{2} .
$$

This structure is identical to that obtained in the massless limit [2], with the integrals $I_{a}$ and $I_{b}$ given by

$$
I_{a}=\int_{p} f(p)\left(1+f(p), \quad I_{b}=\int_{p} \frac{2 f(p)}{p} .\right.
$$

We shall end this section by making a general comment on the Fokker-Planck equation in this massless limit, in order to clarify the interpretation of the two competing terms that are present in the current. For simplicity we focus on the isotropic case so that the kinetic equation is of the form ${ }^{2}$

$$
\partial_{t} f(p)=\frac{1}{p^{2}} \partial_{p}\left\{p^{2}\left[I_{a} \partial_{p} f(p)+I_{b} f(1+f)\right]\right\} .
$$

Note that energy conservation, $\partial_{t} \int_{\boldsymbol{p}} p f(p)=0$, entails

$$
I_{a} \int_{p} \frac{\partial f}{\partial p}=I_{b} \int_{p} f(1+f)
$$

a relation that is obviously satisfied given the definitions (32) of $I_{a}$ and $I_{b}$.

To gain insight into the physical meaning of the two terms on the r.h.s. of this equation, let us multiply both sides by $p^{2}$ and integrate over momentum. We obtain

$$
\partial_{t} \int_{\boldsymbol{p}} p^{2} f(p)=\int_{\boldsymbol{p}} p^{2} \frac{1}{p^{2}} \partial_{p}\left\{p^{2}\left[I_{a} \partial_{p} f(p)+I_{b} f(1+f)\right]\right\} .
$$

\footnotetext{
${ }^{2}$ We absorb here the constant factor $36 \pi \alpha_{s}^{2} \mathcal{L}$ into the redefinition of time (see Eq. (38) below).
} 
Using integration by part for the r.h.s., we eventually obtain

$$
\partial_{t}\left\langle p^{2}\right\rangle=6 I_{a}\langle n\rangle-2 I_{b}\langle\tilde{p}\rangle,
$$

where

$$
\left\langle p^{2}\right\rangle \equiv \int_{\boldsymbol{p}} p^{2} f(p), \quad\langle n\rangle \equiv \int_{\boldsymbol{p}} f(p), \quad\langle\tilde{p}\rangle \equiv \int_{\boldsymbol{p}} p f(1+f) .
$$

The equation (36) may be interpreted as follows. The first term on the r.h.s., $6 I_{a}\langle n\rangle$, represents the diffusion of particles in momentum space that results from multiple small-angle scatterings. If this first term was the only one, the system would diffuse indefinitely, with $\left\langle p^{2}\right\rangle \sim I_{a}\langle n\rangle t$ at late time. The second term on the r.h.s., $-2 I_{b}\langle\tilde{p}\rangle$, represents a drag force, with the characteristic of a friction. It is negative as long as there is any nonzero momentum in the system and therefore opposes the diffusive contribution, which is generically positive. In the absence of diffusion, this term would cause the magnitude of the momentum to decrease continuously, eventually bringing all particles to a state of zero momentum. This drag term is the agent that drives the system towards condensation. Finally, with both terms present on the r.h.s of the equation, thermal equilibrium can be reached, and the system approaches the fixed point corresponding to a Bose-Einstein distribution, with $T=I_{a} / I_{b}$.

\section{Numerical solutions to the transport equation}

We turn now to the discussion of results obtained by solving numerically the Fokker-Plank equation for relevant cases. Details on the numerical procedure that we used can be found in Appendix B. We shall write the transport equation in terms of dimensionless quantities. In order to do so, we express all momenta (and masses, energies, temperature) in units of $Q_{s}$, and the time in units of $1 / Q_{s}$. In fact, we also absorb into the dimensionless time a numerical factor, setting

$$
t \equiv\left(\frac{1}{Q_{s}}\right) \frac{\tau \pi}{18 \alpha_{s}^{2}} .
$$

This is the factor that should be used if one wants to relate the time $\tau$ of the simulation to the physical time $t .{ }^{3}$

In the dimensionless variables, the transport equation reads

$$
\mathcal{D}_{\tau} f(p)=-\frac{1}{p^{2}} \partial_{p}\left\{p^{2} \mathcal{J}(p)\right\}, \quad \mathcal{J}\left(p_{1}\right)=\int_{\boldsymbol{p}_{2}}\left(h_{1} f_{2}^{\prime}-h_{2} f_{1}^{\prime}\right) \mathcal{Z}\left(v_{1}, v_{2}\right) .
$$

Note that since from now on we shall be dealing mostly with dimensionless variables, we keep the same names for the dimensionful and dimensionless momenta.

\footnotetext{
${ }^{3}$ Note that this factor differs by a factor $2 \pi^{2} \mathcal{L}$ from the "natural" factor that appears for instance in the expression $(29)$ of the current. Note that $\pi /\left(18 \alpha_{s}^{2}\right) \approx 0.7$ so that $t \approx \tau / Q_{s}$.
} 

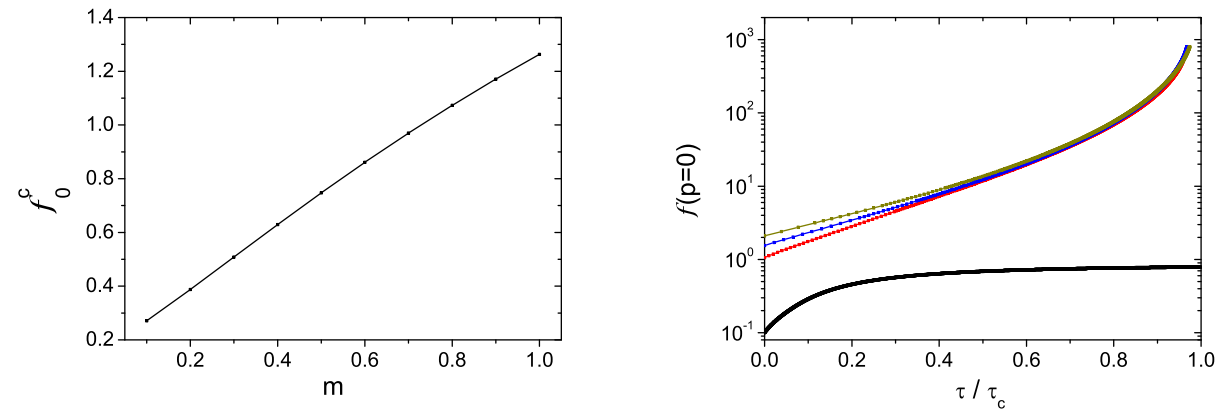

Figure 1: (Color online.) Left: Critical $f_{0}$ as a function of the gluon mass $m$. Points above the curve correspond to overpopulated initial conditions, below the curve to underpopulated initial conditions. Right: The evolution of $f(p \approx 0)$ for different initial occupations $f_{0}$ (with $m=0.3$ ): the lower (black) curve is for underpopulated initial condition, and the other curves are for overpopulated initial conditions.

The initial condition is chosen be of the Glasma type [1], i.e. $f(E)=$ $f_{0}\left(1+e^{10(E-1.5)}\right)^{-1}$, with $E=\sqrt{p^{2}+m^{2}}$. For this family of initial conditions, there is a critical value of $f_{0}$, that we call $f_{0}^{c}$, above which the system becomes overpopulated. When $f_{0}=f_{0}^{c}$, the equilibrium distribution is a BoseEinstein distribution with a maximal chemical potential $\mu=m$. The value of $f_{0}^{c}$ depends on the mass $m$ of the gluons, and its variation with $m$ is illustrated in Fig. 1. It is seen that $f_{0}^{c}$ increases with $m$ : the value of $f_{0}$ required to reach the onset of condensation is larger for massive particles than for massless ones. The right hand panel of Fig. 1 illustrates the behavior of $f(p=0)$ in the two generic situations of underpopulation where $f(0)$ reaches a constant value and that of overpopulation where $f(0)$ diverges at a finite time $\tau=\tau_{c}$.

Note that in all cases, the infrared part of the distribution function evolves rapidly towards an approximate classical thermal distribution function,

$$
f(E) \rightarrow \frac{T^{*}}{\left(E-\mu^{*}\right)}
$$

with an effective temperature $T^{*}$ and effective chemical potential $\mu^{*}$ that can be determined numerically. The evolution of $T^{*}$ and $\mu^{*}$ with time depends on whether the system is over or underpopulated, as we shall discuss in the following.

\subsection{Underpopulated case}

Starting from the initial distribution corresponding to underpopulation, we verify that the system evolves to the expected equilibrium state. The approach to thermalization is illustrated in Fig.2 which displays the evolution of the distribution function $f(p)$ and that of the current $\mathcal{J}(p)$. The current changes its 

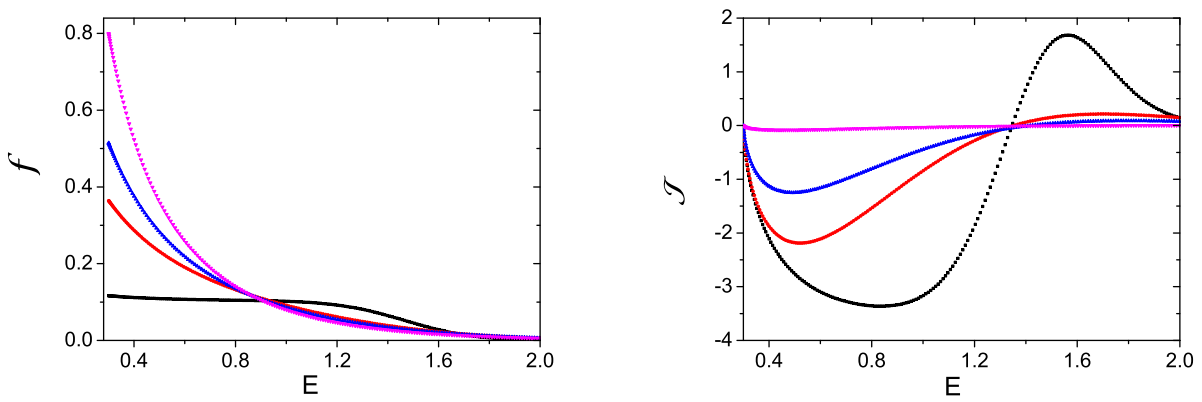

Figure 2: Left: Distribution function $f(E)$ at various times $\tau=0.05,0.8,1.4,6.25$ (from bottom to top at $E=0.4$ ). Right: Current $\mathcal{J}$ at $\tau=0.05,0.8,1.4,6.25$ (from bottom to top at $E=0.4$ ). Underpopulated initial condition, with $m=0.3$ and $f_{0}=0.1$.

sign around $E \simeq 1.3$, the point which separates the effects of the two competing components of the current. For larger values of $E$, the current is diffusive and positive. For smaller values of $E$, the current is dominated by its drag component, and is negative. As time progresses these two components of the current move particles in momentum space, the drag term pushing particles towards small momenta, the diffusion term smoothening the distribution at large momenta. The resulting distribution gradually evolves towards the equilibrium distribution, as indicated in the left panel of Fig. 2, while the current diminishes, and eventually vanishes (which takes place approximately for the largest time considered in the plot). The evolution with time of the effective parameters that characterize the infrared part of the distribution function are displayed in Fig. 3. This figure clearly demonstrates that the system thermalizes as expected.
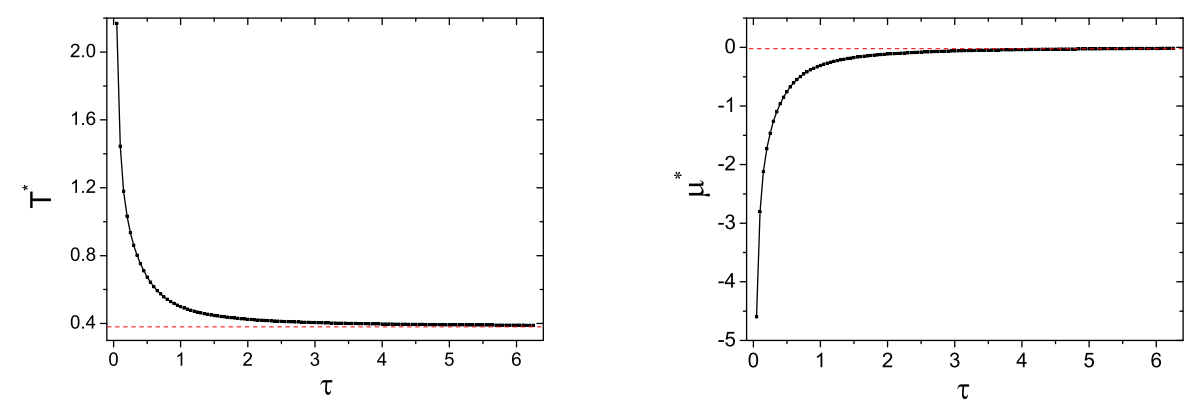

Figure 3: (Color online.) The evolution with time of the effective local temperature $T^{*}$ (left) and chemical potential $\mu^{*}$ (right) for an underpopulated initial condition $(m=0.3$ and $f_{0}=0.1$ ). At late time, both quantities approach their expected equilibrium values, indicated by the horizontal (red) dotted lines. 


\subsection{Overpopulated cases}

We turn now to the situation with overpopulation. We expect, from our previous work, that the system will approach the onset of condensation in a finite time $\tau=\tau_{c}$. We have studied the evolution with different $\operatorname{masses}(m=$ $0.1,0.3,0.5,0.7)$. In the numerical solution, the time step is self-adaptive i.e. becoming smaller when the evolution becomes faster, which allows us to evolve the system in each case very close to the onset. The evolution is stopped when $f>800$ for the smallest momentum grid point, which roughly corresponds to the situation when the difference between the local chemical potential $\mu^{*}$ and the mass becomes less than the mesh size. That is, the evolution is stopped very near the onset of condensation where $\mu=m$. An illustration of the energy dependence of the distribution near the onset is displayed in Fig. 4

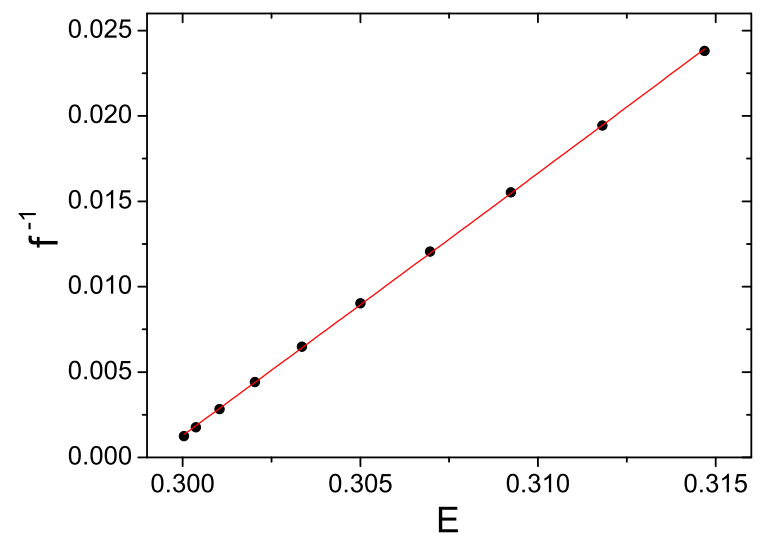

Figure 4: The inverse of the distribution as a function of energy, $1 / f$ versus $E$, for $m=0.3$, and $\tau=0.101$ corresponding to the onset of condensation.

The Table 1 gives some typical values of the parameters $f_{0}^{c}$ and $\tau_{c}$ for different initial conditions, and different values of the mass $m$ and the Debye mass $m_{D}$. One notices that, for fixed masses (e.g. $\left.m=0.3\right)$ the onset time decreases with increasing $f_{0}$, which is as expected: the larger the overpopulation, the shorter time it takes to reach the onset of BEC. For fixed $m$ and $f_{0}$ an increase of the Debye mass leads to an increase of $\tau_{c}$ (compare for instance the lines corresponding to $m=0.7$ and $f_{0}=1$ ). This is because an increase of $m_{D}$ reduces the scattering rate, which slows down the evolution. A larger increase of $\tau_{c}$ results from the increase of $m$ at fixed $f_{0}$ and $m_{D}$. The latter phenomenon is in line with what we observed earlier, namely that $f_{0}^{c}$ increases with $m$.

Different from the underpopulated case, the evolution of the flow of particles is quite different in the overpopulated case, as shown in Fig.5. The negative infrared flux keeps increasing in a roughly self-similar manner. The local chemical potential $\mu^{*}$ approaches $\mu_{c}=m$ at the onset. We have extracted the $\mu^{*}$ 


\begin{tabular}{ccccc}
\hline $\mathrm{m}$ & $m_{D}$ & $f_{0}$ & $f_{0}^{c}$ & $\tau_{c}$ \\
\hline 0.1 & 0.1 & 1.0 & 0.271 & 0.029 \\
0.3 & 0.3 & 1.0 & 0.508 & 0.101 \\
0.5 & 0.5 & 1.0 & 0.747 & 0.259 \\
0.7 & 0.7 & 1.0 & 0.969 & 0.663 \\
0.3 & 0.3 & 1.2 & 0.508 & 0.065 \\
0.3 & 0.3 & 1.4 & 0.508 & 0.046 \\
0.3 & 0.3 & 1.6 & 0.508 & 0.034 \\
0.3 & 0.3 & 1.8 & 0.508 & 0.026 \\
0.3 & 0.3 & 2.0 & 0.508 & 0.021 \\
0.1 & 0.7 & 1.0 & 0.271 & 0.095 \\
0.7 & 0.1 & 1.0 & 0.969 & 0.242 \\
\hline
\end{tabular}

Table 1: Critical occupation factor $f_{0}^{c}$ and onset time scale $\tau_{c}$ for different combinations of parameters.
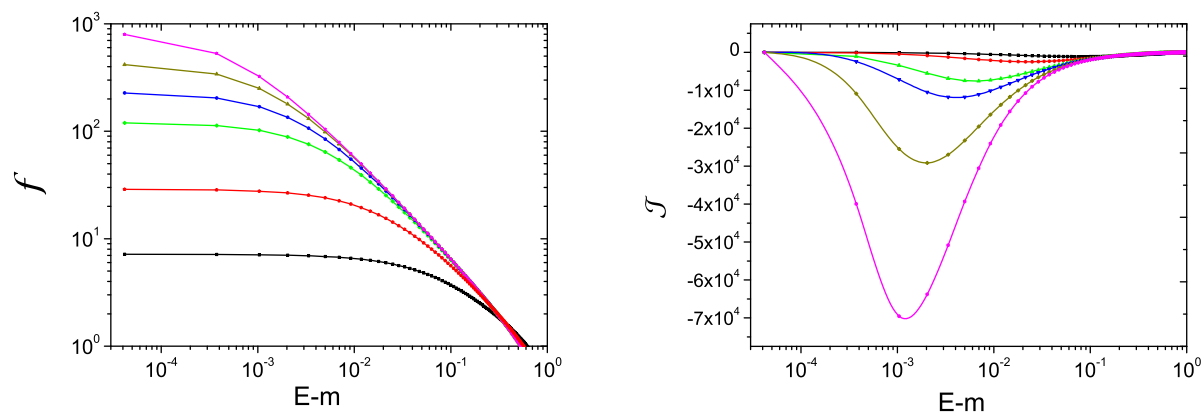

Figure 5: Color online. Distribution function $f$ (left) and current $[\mathcal{J}]$ (right), the curves corresponding, from bottom to top, to increasing values of $\tau: \tau=0.04$ (black), 0.0674(red), 0.0871 (green), 0.0927 (blue), 0.0961 (yellow), 0.0977 (pink) with $m=0.3$ and $f_{0}=1.0$ (overpopulated initial condition).

as a function of time for a variety of mass values as well as initial occupation values: see Fig.6. In all cases, we have found that the evolution of $\mu^{*}$ close to the onset point can be fitted with power law $\mu=m-\lambda\left(\tau_{c}-\tau\right)^{\eta}$. Furthermore in all cases, we've found that the optimal exponent is about one, i.e. $\eta \simeq 1$ ( see also Fig.A.8 of Appendix A.4 for a clearer illustration of this linear scaling relation). This dynamical behavior in the present massive case is essentially the same as that found for the massless case in [2]. It may be noted that the medium-generated mass could become time-dependent in principle which might influence the time evolution of $\mu^{*}$. An explicit check was performed, by determining the mass from the distribution function at each time moment. It is found that the scaling behavior of $\mu^{*}$, approaching onset linearly in time, re- 

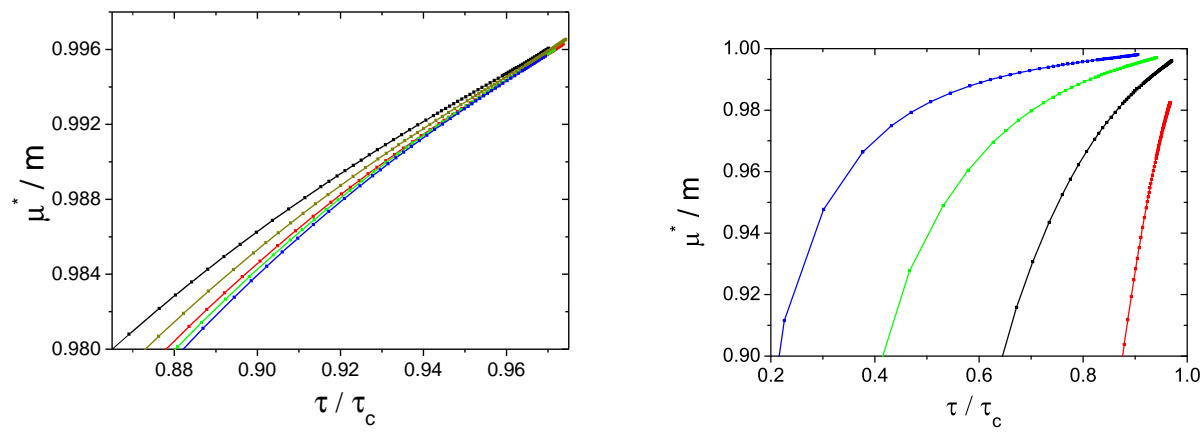

Figure 6: Evolution of IR local chemical potential $\mu^{*}$ toward onset $\mu^{*} \rightarrow m$ for different choices of $f_{0}=1.0,1.2,1.4,1.6,1.8$ (from left to right) with the same mass $m=0.3$. Right: Evolution of IR local chemical potential $\mu^{*}$ toward onset $\mu^{*} \rightarrow m$ for different choices of external mass $m=0.7,0.5,0.3,0.1$ (from left to right) with the same $f_{0}=1$.

mains unchanged. This may be understood from the fact that the mass is a smooth function of time, with no singularity at the onset. Near the onset it is therefore expected to vary linearly with $\tau_{c}-\tau$, which does not affect the linear behavior of $\mu-\mu_{c}$. We give a more complete discussion of the critical regime in the next section.

\section{Critical scaling analysis}

In this section we examine how the scaling behavior that holds near the onset of BEC is affected by the gluon mass. A major modification, with respect to the massless case, concerns the dispersion relation near the onset. When $m \neq 0$, condensation occurs when $m=\mu$, and the dispersion relation becomes non relativistic, $E_{p}-\mu=\sqrt{p^{2}+m^{2}}-m \approx p^{2} /(2 m)$, which differs from the ultra relativistic dispersion relation of the massless case, $E_{p}=p$, where condensation occurs at $\mu=0$. This change in the dispersion relation modifies the singularity near the onset, but, as we shall see, this does not affect in a major way the onset critical behavior. The foregoing analysis follows closely that presented in Ref. [2].

\subsection{Scaling behavior of the current at small momentum}

As we have seen in solving numerically the Fokker-Planck equation, for generic initial conditions, the distribution function at small momentum evolves rapidly towards the approximate equilibrium distribution:

$$
f(p) \approx \frac{T^{*}}{E(p)-\mu^{*}} \approx \frac{2 m T^{*}}{p^{2}+2 m \delta \mu}=\frac{2 m T^{*}}{p^{2}+\Delta^{2}},
$$

where we have set $\delta \mu \equiv m-\mu^{*}$ and $\Delta \equiv \sqrt{2 m \delta \mu}$. As the system approaches condensation, $\Delta \rightarrow 0$. To analyze the detailed behavior of the properties of the 
system when $\Delta \rightarrow 0$, it is convenient to calculate the time dependence of the number of particles inside a small sphere of radius $p_{0}$ centered at the origin. In doing this calculation, we assume that $f(p)$ keeps the form (41), with time dependent parameters $T^{*}$ and $\delta \mu$. In particular, $f(0)$ remains finite as long as $\delta \mu \neq 0$. A simple calculation then yields

$$
\partial_{\tau} \int_{0}^{p_{0}} d p p^{2} f(p)=2 m \Delta\left(\partial_{\tau} T^{*}\right) h_{1}(y)+2 m T^{*}\left(\partial_{\tau} \Delta\right) h_{2}(y)
$$

where $y \equiv p_{0} / \Delta$, and the two scaling functions are

$$
\begin{aligned}
& h_{1}(y)=y-\operatorname{ArcTan}(y) \\
& h_{2}(y)=\frac{y}{1+y^{2}}-\operatorname{ArcTan}(y)
\end{aligned}
$$

By using Eq. (28), one can relate the left hand side of Eq. (42) to the current at $p_{0}$, and obtain

$$
-\mathcal{J}\left(p_{0}\right)=\frac{\partial_{\tau} \int_{0}^{p_{0}} d p p^{2} f(p)}{p_{0}^{2}}=\frac{2 m\left(\partial_{\tau} T^{*}\right)}{\Delta} \frac{h_{1}(y)}{y^{2}}+\frac{2 m T^{*}\left(\partial_{\tau} \Delta\right)}{\Delta^{2}} \frac{h_{2}(y)}{y^{2}}
$$

This equation provides interesting constraints on the small $p$ behavior of $\mathcal{J}(p)$. In the limit $y \rightarrow 0$ or $p_{0} \ll \Delta$, one gets

$$
-\mathcal{J}\left(p_{0}\right) \simeq p_{0} \times \frac{2 m}{3} \partial_{\tau}\left(\frac{T^{*}}{\Delta^{2}}\right)=p_{0} \times \frac{1}{3} \partial_{\tau}\left(\frac{T^{*}}{\delta \mu}\right)=\frac{p_{0}}{3} \partial_{\tau} f(0) .
$$

The current is linear in $p_{0}$, with a slope proportional to $\partial_{\tau} f(0)$, very much like in the massless case [2]. On the other hand, in the limit $y \gg 1$ or $\Delta \ll p_{0} \ll T^{*}$, one obtains the following leading order result ${ }^{4}$

$$
-\mathcal{J}\left(p_{0}\right) \rightarrow \frac{1}{p_{0}^{2}} \times(\pi m) \partial_{\tau}\left(-T^{*} \Delta\right) .
$$

In this region, the current exhibits a singular behavior in $1 / p_{0}^{2}$. The value $(\approx \Delta)$ of the momentum where the change of regime occurs decreases with time, while the absolute value of the current at the minimum becomes larger and larger, and eventually diverges at the onset. This behavior is illustrated in Fig. 7, which shows that the leading qualitative behavior is indeed well captured by Eq. (45). As was the case in the massless case, the current in the scaling regime is dominated by the second term in Eq. (45), which is the one used in the plot of the left panel of Fig. 7. In this plot, we have assumed that the dependence of $m-\mu^{*}$ on $\tau_{c}-\tau$ is linear. A detailed discussion of this point is presented in Appendix Appendix A.4.

\footnotetext{
${ }^{4}$ The subleading contribution is $\sim 1 / p$ and comes from the first term in Eq. (45). It becomes the dominant contribution after onset, when $\delta \mu=0$ and therefore (47) vanishes.
} 

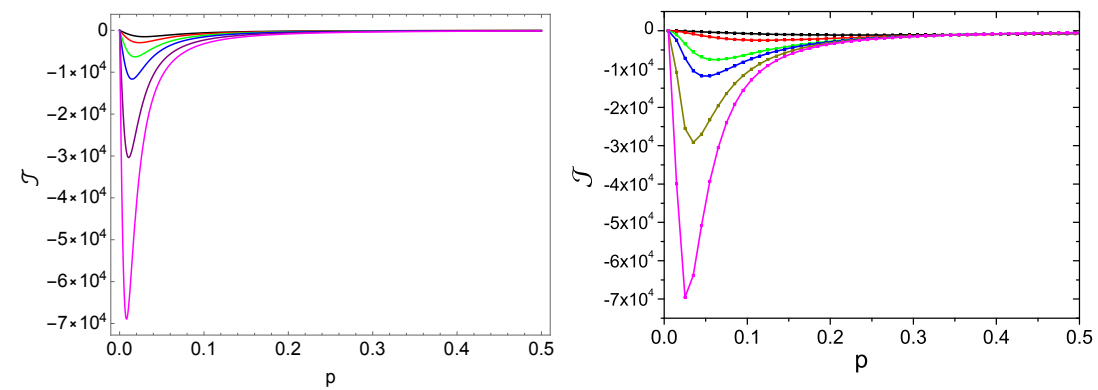

Figure 7: The current (in arbitrary units) as a function of momentum, at different time moments (earlier to later time from top to bottom) close to onset. Left panel: calculated according to the formula (45), assuming a linear relation between $\mu^{*}-m$ and $\tau_{c}-\tau$, and neglecting the time variation of $T^{*}$; right panel: obtained from the numerical solution. The linear behavior at small $p$ followed, as $p$ increases, by a singular behavior in $1 / p^{\alpha}$, with $\alpha>0$ is clearly visible on both figures.

\section{Summary}

In this paper, we have extended the analysis carried out in Ref. [2] to the case where the gluons are given a small mass $m$. The main difference with respect to the massless case studied in [2] is the change of the dispersion relation of the gluon modes, from ultra-relativistic in the massless case to non-relativistic in the massive case. While this change in the dispersion relation leads to an enhanced singularity of the distribution function near the onset of Bose condensation, from $1 / p$ to $1 / p^{2}$, we find that the critical regime that accompanies the approach to the onset is qualitatively unchanged. The Debye mass $m_{D}$ controls the infrared behavior of the collision kernel. It is kept constant, and independent of $m$, although in a more complete theory, both masses are related, and would depend on the temperature. However this is expected to have little impact at the onset, since the onset process is dominated by a critical regime where the actual value of the mass is irrelevant. We have checked in articular that letting the Debye mass adjust with temperature as one approaches BEC does not lead to any significant changes in the behavior of the system. Finally, the presence of the mass $m$ allows us to define properly the equations that governs the dynamics beyond the onset, that is, in the presence of the condensate. This is discussed in a companion paper [17].

\section{Acknowledgements}

The research of JPB is supported by the European Research Council under the Advanced Investigator Grant ERC-AD-267258. That of YJ and JL is supported by the National Science Foundation under Grant No. PHY-1352368. JL is also grateful to the RIKEN BNL Research Center for partial support. 


\section{Appendix A. Derivation of the current $\mathcal{J}(p)$}

The component $i$ of the current $\mathcal{J}\left(\boldsymbol{p}_{1}\right)$ in Eq. (15) can be written

$$
\begin{aligned}
\mathcal{J}^{i}\left(\boldsymbol{p}_{1}\right) & =18 \pi g^{4} \int \frac{\mathrm{d}^{3} \boldsymbol{q}}{(2 \pi)^{3}} \int \frac{\mathrm{d}^{3} \boldsymbol{p}_{2}}{(2 \pi)^{3}}\left(h_{1} f_{2}^{\prime}-h_{2} f_{1}^{\prime}\right) \\
& \times \quad \boldsymbol{q}^{i}\left(\boldsymbol{q} \cdot \boldsymbol{v}_{2}\right) \frac{\left(1-\boldsymbol{v}_{1} \cdot \boldsymbol{v}_{2}\right)^{2}}{\left[\left(\boldsymbol{v}_{1} \cdot \boldsymbol{q}\right)^{2}-\boldsymbol{q}^{2}-m_{D}^{2}\right]^{2}} \delta\left(\boldsymbol{q} \cdot \boldsymbol{v}_{1}-\boldsymbol{q} \cdot \boldsymbol{v}_{2}\right) .
\end{aligned}
$$

Appendix A.1. Details of the angular integration

In order to perform the angular integrals, we choose the following coordinate frames: We define the orientation of $\boldsymbol{q}$ in a frame where $\boldsymbol{p}_{1}$ is along the $\hat{z}$ axis, and denote the corresponding angles by $\theta$ and $\phi$. The orientation of $\boldsymbol{p}_{2}$, given $\boldsymbol{p}_{1}$ and $\boldsymbol{q}$, is defined by the angles $\theta_{2}, \phi_{2}$ in a frame with $\boldsymbol{q}$ along $\hat{z}_{2}$ and $\boldsymbol{q}, \boldsymbol{p}_{1}$ spanning the $\hat{x}_{2}-\hat{z}_{2}$ plane. We have therefore $\boldsymbol{q} \cdot \boldsymbol{v}_{1}=q v_{1} \cos \theta, \boldsymbol{q} \cdot \boldsymbol{v}_{2}=q v_{2} \cos \theta_{2}$, and $\boldsymbol{v}_{1} \cdot \boldsymbol{v}_{2}=v_{1} v_{2}\left(\cos \theta \cos \theta_{2}+\sin \theta \sin \theta_{2} \cos \phi_{2}\right)$.

By integrating over $\phi_{2}$ one gets

$$
\begin{gathered}
\int_{0}^{2 \pi} d \phi_{2}\left(1-\boldsymbol{v}_{1} \cdot \boldsymbol{v}_{2}\right)^{2}=\int_{0}^{2 \pi} d \phi_{2}\left[1-v_{1} v_{2}\left(\cos \theta \cos \theta_{2}+\sin \theta \sin \theta_{2} \cos \phi_{2}\right)\right]^{2} \\
=\pi\left[2\left(1-v_{1} v_{2} \cos \theta \cos \theta_{2}\right)^{2}+v_{1}^{2} v_{2}^{2}\left(1-\cos ^{2} \theta\right)\left(1-\cos ^{2} \theta_{2}\right)\right]
\end{gathered}
$$

Furthermore we rewrite the delta function as

$$
\begin{aligned}
& q \delta\left(\boldsymbol{q} \cdot \boldsymbol{v}_{1}-\boldsymbol{q} \cdot \boldsymbol{v}_{2}\right)=q \delta\left(q\left(v_{1} \cos \theta-v_{2} \cos \theta_{2}\right)\right)=\delta\left(v_{1} \cos \theta-v_{2} \cos \theta_{2}\right) \\
& \quad=\theta\left(v_{1}-v_{2}\right) \delta\left(v_{1} \cos \theta-v_{2} \cos \theta_{2}\right)+\theta\left(v_{2}-v_{1}\right) \delta\left(v_{2} \cos \theta_{2}-v_{1} \cos \theta\right)
\end{aligned}
$$

and change variables, setting $x_{1}=v_{1} \cos \theta, x_{2}=v_{2} \cos \theta_{2}$. One can then complete the angular integration. Note that, by symmetry, the current is aligned on the direction of $\boldsymbol{p}_{1}$, i.e., $\overrightarrow{\mathcal{J}}\left(\boldsymbol{p}_{1}\right)=\hat{\boldsymbol{p}}_{1} \mathcal{J}\left(p_{1}\right)$, with $\mathcal{J}\left(p_{1}\right)$ a function of $p_{1}=\left|\boldsymbol{p}_{1}\right|$ only, and $\hat{\boldsymbol{p}}_{1}=\boldsymbol{p}_{1} / p_{1}$.. We get

$$
\mathcal{J}\left(p_{1}\right)=\frac{18 \alpha_{s}^{2}}{\pi} \int \mathrm{d} p_{2} p_{2}^{2}\left(h_{1} f^{\prime}{ }_{2}-h_{2} f_{1}^{\prime}\right) \int \frac{\mathrm{d} q}{q} \frac{Z\left(v_{1}, v_{2}, c_{q}\right)}{v_{1}},
$$


with

$$
\begin{aligned}
v_{1} v_{2} Z\left(v_{1}, v_{2}, c_{q}\right) & =\frac{1}{2}\left[\int_{-v_{2}}^{v_{2}} \mathrm{~d} x_{2} x_{2}^{2} \frac{2\left(1-x_{2}^{2}\right)^{2}+\left(v_{1}^{2}-x_{2}^{2}\right)\left(v_{2}^{2}-x_{2}^{2}\right)}{\left(x_{2}^{2}-c_{q}\right)^{2}} \theta\left(v_{1}-v_{2}\right)\right. \\
& \left.+\int_{-v_{1}}^{v_{1}} \mathrm{~d} x_{1} x_{1}^{2} \frac{2\left(1-x_{1}^{2}\right)^{2}+\left(v_{1}^{2}-x_{1}^{2}\right)\left(v_{2}^{2}-x_{1}^{2}\right)}{\left(x_{1}^{2}-c_{q}\right)^{2}} \theta\left(v_{2}-v_{1}\right)\right] \\
& =v\left[v^{2}-6\left(1-c_{q}\right)+\left(1-v_{1}^{2}\right)+\left(1-v_{2}^{2}\right)\right. \\
& \left.+\frac{\left(1-c_{q}\right)^{2}}{c_{q}-v^{2}}+\frac{\left(c_{q}-v_{1}^{2}\right)\left(c_{q}-v_{2}^{2}\right)}{2\left(c_{q}-v^{2}\right)}\right] \\
& +\left[-\frac{\left(1-c_{q}\right)^{2}}{c_{q}}-\frac{\left(c_{q}-v_{1}^{2}\right)\left(c_{q}-v_{2}^{2}\right)}{2 c_{q}}-\left(1-v_{1}^{2}\right)-\left(1-v_{2}^{2}\right)\right. \\
& \left.+6\left(1-c_{q}\right)\right] \sqrt{c_{q}} \operatorname{ArcTanh}\left(\frac{v}{\sqrt{c_{q}}}\right),
\end{aligned}
$$

where $c_{q}=1+m_{D}^{2} / q^{2}, v=\min \left(v_{1}, v_{2}\right)$, and we have used the following integral formula to get the final results

$$
\begin{aligned}
& \int \mathrm{d} x x^{2}\left[3 C+\frac{B}{c_{0}-x^{2}}+\frac{A}{\left(c_{0}-x^{2}\right)^{2}}\right] \\
& =C x^{3}-B x-\frac{A x}{2\left(x^{2}-c_{0}\right)}+\left(B \sqrt{c_{0}}-\frac{A}{2 \sqrt{c_{0}}}\right) \operatorname{ArcTanh}\left(\frac{x}{\sqrt{c_{0}}}\right),
\end{aligned}
$$

with $A=2\left(c_{0}-1\right)^{2}+\left(c_{0}-v_{1}^{2}\right)\left(c_{0}-v_{2}^{2}\right), B=4+v_{1}^{2}+v_{2}^{2}-6 c_{0}, C=1$.

\section{Appendix A.2. Limiting cases}

There are a few limiting cases where the expression above simplifies. For instance, in the limit of vanishing screening mass $m_{D} \rightarrow 0, c_{q}=1$ and

$$
\begin{aligned}
v_{1} v_{2} Z & =v\left[v^{2}+\left(1-v_{1}^{2}\right)+\left(1-v_{2}^{2}\right)-\frac{\left(1-v_{1}^{2}\right)\left(1-v_{2}^{2}\right)}{2\left(1-v^{2}\right)}\right] \\
& +\left[\frac{\left(1-v_{1}^{2}\right)\left(1-v_{2}^{2}\right)}{2}-\left(1-v_{1}^{2}\right)-\left(1-v_{2}^{2}\right)\right] \operatorname{ArcTanh}(v)
\end{aligned}
$$

In this limit, the remaining $q$-integration in the expression (A.4) of the current becomes simply the usual Coulomb logarithm, $\int d q / q \rightarrow \mathcal{L}$.

In the limit where all effective masses vanish, i.e. $m \rightarrow 0$ and $m_{D} \rightarrow 0, c_{q}$ and all the velocities become unity, $v_{1} v_{2} Z \rightarrow 1$ and we recover the expression of the massless case studied in Ref. [2].

In the limit where the colliding gluons become massless, i.e., $m \rightarrow 0$, but the 
Debye mass stays finite, all the velocities $v_{1}, v_{2}, v$ approach unity, and we get

$$
\begin{aligned}
v_{1} v_{2} Z= & {\left[1-\frac{15}{2}\left(1-c_{q}\right)\right] } \\
& +\sqrt{c_{q}}\left(1-c_{q}\right)\left[-\frac{3\left(1-c_{q}\right)}{2 c_{q}}+6\right] \operatorname{ArcTanh}\left(\frac{1}{\sqrt{c_{q}}}\right) .
\end{aligned}
$$

This expression is finite when $q \rightarrow 0$. To see that, recall that when $q \rightarrow 0$, $c_{q} \sim m_{D}^{2} / q^{2} \rightarrow \infty$. As simple calculation then yields

$$
v_{1} v_{2} Z \approx A c_{q}^{-2}+\mathcal{O}\left(c_{q}^{-3}\right),
$$

where

$$
\left.A=\frac{3}{7} v^{7}-\frac{v^{5}}{5}\left(4+v_{1}^{2}+v_{2}^{2}\right)+\frac{v^{3}}{3}\left(2+v_{1}^{2} v_{2}^{2}\right)\right] .
$$

This result confirms that in the presence of a non-vanishing screening mass $m_{D}$, the function $Z$ is regular as $q \rightarrow 0$, and the $q$-integration in the expression (A.4) becomes infrared finite, as expected.

\section{Appendix A.3. Detailed derivation of the q-integration}

In the general case, in order to perform the $q$-integration in Eq. (A.4), we rewrite $v_{1} v_{2} Z$ in Eq. (A.5) as follows:

$$
\begin{aligned}
v_{1} v_{2} Z\left(c_{q}, v_{1}, v_{2}\right)= & \frac{E_{1}}{c_{q}-v^{2}}+E_{2}\left(c_{q}-v^{2}\right)+E_{3}+\sqrt{c_{q}} \operatorname{ArcTanh}\left(\frac{v}{\sqrt{c_{q}}}\right) \\
& \times\left[E_{4}\left(1-c_{q}\right)+E_{5} \frac{c_{q}-1}{c_{q}}+E_{6}\right],
\end{aligned}
$$

where

$$
\begin{aligned}
& E_{1}=\frac{v}{4}\left[2 v^{2}\left(3 v^{2}-v_{1}^{2}-v_{2}^{2}-4\right)+2 v_{1}^{2} v_{2}^{2}+4\right], \quad E_{2}=\frac{15 v}{2} \\
& E_{3}=\frac{v}{2}\left(20 v^{2}-3 v_{1}^{2}-3 v_{2}^{2}-12\right), \quad E_{4}=\frac{15}{2} \\
& E_{5}=\frac{1}{4}\left[-2 v_{1}^{2} v_{2}^{2}-4\right], \quad E_{6}=\frac{1}{4}\left[-2 v_{1}^{2} v_{2}^{2}+6 v_{1}^{2}+6 v_{2}^{2}-10\right] .
\end{aligned}
$$

Then, we change integration variable from $q$ to $c_{q}$ and obtain

$$
\begin{aligned}
& \int \frac{d q}{q} v_{1} v_{2} Z\left(q, v_{1}, v_{2}\right)=\int \frac{d c_{q}}{2\left(1-c_{q}\right)} v_{1} v_{2} Z\left(c_{q}, v_{1}, v_{2}\right) \\
& =E_{1} G_{1}\left(v, c_{q}\right)+E_{1} G_{1}\left(v, c_{q}\right)+E_{2} G_{2}\left(v, c_{q}\right)+E_{3} G_{3}\left(v, c_{q}\right) \\
& +E_{4} G_{4}\left(v, c_{q}\right)+E_{5} G_{5}\left(v, c_{q}\right)+E_{6} G_{6}\left(v, c_{q}\right) .
\end{aligned}
$$


The results of these integrals are listed below:

$$
\begin{aligned}
& G_{1}\left(v, c_{q}\right)=\int d c_{q} \frac{1}{2\left(1-c_{q}\right)\left(c_{q}-v^{2}\right)}=-\frac{\ln \left(c_{q}-v^{2}\right)-\ln \left(c_{q}-1\right)}{2\left(v^{2}-1\right)} \\
& G_{2}\left(v, c_{q}\right)=\int d c_{q} \frac{c_{q}-v^{2}}{2\left(1-c_{q}\right)}=-\frac{1}{2}\left[c_{q}+\left(1-v^{2}\right) \ln \left(c_{q}-1\right)\right] \\
& G_{3}\left(v, c_{q}\right)=\int d c_{q} \frac{1}{2\left(1-c_{q}\right)}=-\frac{1}{2} \ln \left(c_{q}-1\right) \\
& G_{4}\left(v, c_{q}\right)=\int d c_{q} \frac{1}{2} \sqrt{c_{q}} \operatorname{ArcTanh}\left(\frac{v}{\sqrt{c_{q}}}\right)=\frac{1}{6}\left[2 c_{q}^{3 / 2} \operatorname{ArcTanh}\left(\frac{v}{\sqrt{c_{q}}}\right)+v^{3} \ln \left(c_{q}-v^{2}\right)+c_{q} v\right] \\
& G_{5}\left(v, c_{q}\right)=\int d c_{q} \frac{1}{2 c_{q}} \operatorname{ArcTanh}\left(\frac{v}{\sqrt{c_{q}}}\right)=\frac{1}{2}\left[v \ln \left(c_{q}-v^{2}\right)+2 \sqrt{c_{q}} \operatorname{ArcTanh}\left(\frac{v}{\sqrt{c_{q}}}\right)\right] \\
& G_{6}\left(v, c_{q}\right)=\int d c_{q} \frac{1}{2\left(1-c_{q}\right)} \operatorname{ArcTanh}\left(\frac{v}{\sqrt{c_{q}}}\right) \\
& =v\left\{-\frac{\sqrt{c_{q}}}{v} \operatorname{ArcTanh}\left(\frac{v}{\sqrt{c_{q}}}\right)+\ln \left(\frac{v}{\sqrt{c_{q}}}\right)-\frac{1}{2} \ln \left(1-\frac{v^{2}}{c_{q}}\right)\right. \\
& -\frac{1}{4 v}\left[2 \ln \left(\frac{\sqrt{c_{q}}-1}{\sqrt{c_{q}}+1}\right) \operatorname{ArcTanh}\left(\frac{v}{\sqrt{c_{q}}}\right)+\operatorname{Li} \operatorname{Ti}_{2}\left(\frac{1-v / \sqrt{c_{q}}}{1+v}\right)+\operatorname{Li}_{2}\left(-\frac{v+v \sqrt{c_{q}}}{1-v}\right)\right. \\
& +\operatorname{Li}{ }_{2}\left(-\frac{v-v \sqrt{c_{q}}}{1-v}\right)+\operatorname{Li}{ }_{2}\left(\frac{1+v / \sqrt{c_{q}}}{1+v}\right) \\
& +\ln \left(v-v / \sqrt{c_{q}}\right) \ln \left(1-v / \sqrt{c_{q}}\right)-\ln \left(v^{2}-v^{2} / c_{q}\right) \ln (1-v) \\
& \left.\left.-\ln \left(1-v^{2} / c_{q}\right) \ln (1+v)+\ln \left(v+v / \sqrt{c_{q}}\right) \ln \left(1+v / \sqrt{c_{q}}\right)\right]\right\} .
\end{aligned}
$$

We define, with $\Lambda$ an ultraviolet cutoff ${ }^{5}$ :

$$
\int_{0}^{\Lambda} \frac{d q}{q} v_{1} v_{2} Z\left(c_{q}, v_{1}, v_{2}\right)=L\left(c_{\Lambda}, v_{1}, v_{2}\right)-L\left(\infty, v_{1}, v_{2}\right),
$$

where the first argument of $L$ denotes the value of $c_{q}$ (when $q=\Lambda$ or $q=0$, respectively). As we have already shown, there is no singularity at $q \rightarrow 0$ i.e. $c_{q} \rightarrow \infty$ when $m_{D}$ is finite, and we have the following finite result

$$
\begin{aligned}
& L\left(\infty, v_{1}, v_{2}\right)=\frac{1}{24}\left\{20 v^{3}-12 v\left(v_{1}^{2} v_{2}^{2}+2\right)\right] \\
& \left.-3 \Delta\left[\operatorname{Li}_{2}\left(\frac{v}{v-1}\right)+\operatorname{Li}_{2}\left(\frac{1}{v+1}\right)+2 v-(2 v+\ln (1-v)) \ln (v)\right]\right\}
\end{aligned}
$$

\footnotetext{
${ }^{5}$ One expects $\Lambda$ to be typically of the order of the temperature. The numerical calculations have been performed with a somewhat larger value, $\Lambda=4 Q_{s}$.
} 
where $\Delta=-2 v_{1}^{2} v_{2}^{2}+6\left(v_{1}^{2}+v_{2}^{2}\right)-10$. Note in the massless limit for external gluons, i.e. $m \rightarrow 0$, one has $\Delta \rightarrow 0$ and the expression above greatly simplifies:

$$
L\left(c_{\Lambda}\right)-L(\infty)=-\frac{1}{2}\left[5 c_{\Lambda}+\left(3-5 c_{\Lambda}\right) \sqrt{c_{\Lambda}} \operatorname{Arc} \operatorname{Coth}\left(\sqrt{c_{\Lambda}}\right)-\frac{4}{3}\right] .
$$

We write the final result for the current in the form

$$
\mathcal{J}\left(\boldsymbol{p}_{1}\right)=\frac{18 \alpha_{s}^{2}}{\pi} \int d p_{2} p_{2}^{2} \mathcal{Z}\left(v_{1}, v_{2}\right)\left[h_{1} f_{2}^{\prime}-h_{2} f_{1}^{\prime}\right]
$$

with

$$
\begin{aligned}
\mathcal{Z}\left(v_{1}, v_{2}\right) & =\int \frac{\mathrm{d} q}{q} \frac{Z\left(v_{1}, v_{2}, c_{q}\right)}{v_{1}} \\
& =\left(v_{1}^{2} v_{2}\right)^{-1}\left[L\left(c_{\Lambda}, v_{1}, v_{2}\right)-L\left(\infty, v_{1}, v_{2}\right)\right]
\end{aligned}
$$

\section{Appendix A.4. The small momentum regime}

We now analyze the small momentum form of the current, $\mathcal{J}\left(p_{1} \rightarrow 0\right)$. The leading order expression of $\mathcal{Z}$ in the limit $v_{1} \rightarrow 0$ can be obtained after a lengthy but straightforward calculation. It reads

$$
\mathcal{Z}\left(v_{1} \rightarrow 0, v_{2}\right) \approx \mathcal{L} \frac{v^{3}}{v_{1}^{2} v_{2}}
$$

where $v=\min \left(v_{1}, v_{2}\right)$, and $\mathcal{L}$ is a positive constant,

$$
\mathcal{L}=-\frac{1}{3}\left[\log \left(c_{\Lambda}-1\right)-\log \left(c_{\Lambda}\right)+\frac{1}{c_{\Lambda}}\right], \quad c_{\Lambda}=1+\frac{m_{D}^{2}}{\Lambda^{2}} .
$$

The small momentum current can therefore be written $\operatorname{as}^{6}$

$$
-\mathcal{J}\left(p_{1}\right)=36 \pi \alpha_{s}^{2} \mathcal{L} \int_{\boldsymbol{p}_{2}} \frac{v^{3}}{v_{1}^{2} v_{2}}\left[h_{2} f_{1}^{\prime}-h_{1} f_{2}^{\prime}\right] .
$$

Since the numerical factor in front of the integral is to be absorbed in the redefinition of the time scale (see Eq. (38)), we rewrite $\mathcal{J}\left(p_{1}\right)$ as

$$
-\mathcal{J}\left(p_{1}\right)=I_{a}\left(p_{1}\right) f_{1}^{\prime}+I_{b}\left(p_{1}\right) h_{1},
$$

where

$$
I_{a}\left(p_{1}\right)=\int_{p_{2}} h_{2} \frac{v^{3}}{v_{1}^{2} v_{2}}, \quad I_{b}\left(p_{1}\right)=-\int_{p_{2}} f_{2}^{\prime} \frac{v^{3}}{v_{1}^{2} v_{2}}
$$

\footnotetext{
${ }^{6}$ It is not difficult to show that, with this approximate expression for the current, particle number as well as energy are conserved.
} 
These integrals reduce to the integrals $I_{a}$ and $I_{b}$ of Eq. (32) in the massless limit (in this limit, the dependence on $p_{1}$ disappears and $\mathcal{L}$ becomes the usual Coulomb logarithm).

We argue in the main text, and we have verified through numerical calculations, that at small momentum and near the onset for BEC, the distribution function $f(p)$ can be well approximated by a Bose equilibrium distribution function We therefore write the distribution in the momentum range of interest $(p \ll \Delta=\sqrt{2 m \delta \mu})$ as

$$
f(p)=f^{*}(p)+\delta f(p),
$$

where $f^{*}(p)$ is an equilibrium Bose distribution with temperature $T^{*}$ and chemical potential $\mu^{*}$, and $\delta f(p)$ represents a small deviation from this equilibrium distribution. We assume that $\delta f(p)$ is a regular function of $p$ at $p=0$.

In order to calculate the integrals $I_{a, b}$ we need to pay attention to the fact that $v=\min \left(v_{1}, v_{2}\right)$, and divide the $p_{2}$ integration range appropriately, making explicit the dependence on $p_{1}$. We obtain

$$
\begin{aligned}
2 \pi^{2} I_{a}\left(p_{1}\right) & =\int_{0}^{p_{1}} d p_{2} p_{2}^{2} \frac{v_{2}^{2}}{v_{1}^{2}} h_{2}+\int_{p_{1}}^{\infty} d p_{2} p_{2}^{2} \frac{v_{1}}{v_{2}} h_{2} \\
& =\int_{0}^{p_{1}} d p_{2} p_{2}^{2}\left(\frac{v_{2}^{2}}{v_{1}^{2}}-\frac{v_{1}}{v_{2}}\right) h_{2}+\int_{0}^{\infty} d p_{2} p_{2}^{2} \frac{v_{1}}{v_{2}} h_{2} \\
& =\int_{0}^{p_{1}} d p_{2} p_{2}^{2}\left(\frac{p_{2}^{2}}{p_{1}^{2}}-\frac{p_{1}}{p_{2}}\right) h_{2}+\frac{p_{1}}{m} \int_{0}^{\infty} d p_{2} p_{2} E_{2} h_{2} .
\end{aligned}
$$

In the first integral, we could set $v_{1} \approx p_{1} / m$ and $v_{2}=p_{2} / m$, while in the second integral we need to keep the exact expression $v_{2}=p_{2} / E_{2}$. Since, when $p_{1} \ll \sqrt{2 m \delta \mu}, h_{2}$ in the first integral is nearly a constant, this first integral is of order $p_{1}^{3}$, and is therefore negligible compared to the second one $\propto p_{1} / m$. We are then left with the integral given in Eq. (31). Proceeding in the same way for $-I_{b}$, one obtains an identical expression to that of $I_{a}$ with $f_{2}^{\prime}$ substituted to $h_{2}$. We shall denote by $I_{a}^{*}$ and $I_{b}^{*}$, the integrals $I_{a}$ and $I_{b}$ calculated with the distribution $f^{*}$, and write accordingly $I_{a, b}=I_{a, b}^{*}+\delta I_{a, b}$. We may then expand the current (A.24) as follows

$$
-\mathcal{J}\left(p_{1}\right)=\delta I_{a}\left(f_{1}^{*}\right)^{\prime}+\delta I_{b} h_{1}^{*}+I_{a}^{*}\left(\delta f_{1}\right)^{\prime}+I_{b}^{*} \delta h_{1},
$$

where $\delta h_{1} \approx \delta f_{1}\left(1+2 f_{1}^{*}\right)$, and we have used the fact that $I_{a}^{*}=T^{*} I_{b}^{*}$ which follows immediately from the relation $\left(f^{*}\right)^{\prime}=-\left(1 / T^{*}\right) h^{*}$. By using again the same identity for the first two terms of the equation above, we can rewrite the current as

$$
\mathcal{J}\left(p_{1}\right)=\frac{1}{T^{*}}\left(\delta I_{a}-T^{*} \delta I_{b}\right) h_{1}^{*}-I_{a}^{*}\left(\delta f_{1}\right)^{\prime}-I_{b}^{*} \delta h_{1} .
$$

At this point, we note that the last two terms in the expression above can be neglected as $p_{1} \rightarrow 0$. Indeed, $\delta f\left(p_{1}\right)$ is regular as $p_{1} \rightarrow 0$, while $h_{1}^{*} \sim\left(f_{1}^{*}\right)^{2}$ 
diverges $\left(\delta h_{1} \sim \delta f_{1} f_{1}^{*}\right.$ is subleading). A simple calculation yields

$$
\delta \tilde{I}_{a}-\delta \tilde{I}_{b} T^{*} \approx \frac{p_{1}}{m} \int_{0}^{\infty} d p_{2} p_{2} E_{2}\left[\delta f_{2}\left(1+2 f_{2}^{*}\right)+T^{*}\left(\delta f_{2}\right)^{\prime}\right] .
$$

Thus, after dropping the last two terms in Eq. (A.29) one can rewrite $\mathcal{J}$ as follows

$$
\mathcal{J}\left(p_{1}\right)=\frac{1}{3} \gamma(\tau) p_{1} f^{2}\left(p_{1}\right)
$$

where $\gamma(\tau)$ is a priori a regular function of time which can be expanded around the onset time $\tau=\tau_{c}, \gamma(\tau) \simeq \gamma_{c}+\alpha\left(\tau_{c}-\tau\right)$. By inserting this expression in
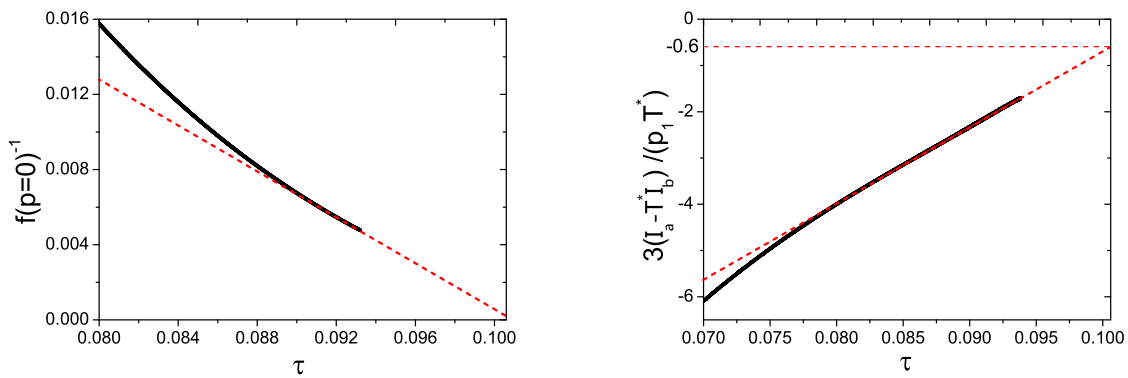

Figure A.8: $f_{p_{1}}^{-1}$ (left) and $\gamma($ right) as function of time with $m=0.3$. Red lines are linear fitting results.

Eq. (46) one gets the following equation for $f(0) \simeq f\left(p_{1}\right)$

$$
\partial_{\tau} f_{p_{1}}^{-1}=\gamma(\tau)
$$

whose solution reads

$$
f_{p_{1}}^{-1}(\tau) \simeq-\gamma_{c}\left(\tau_{c}-\tau\right)-\frac{1}{2} \alpha\left(\tau_{c}-\tau\right)^{2}
$$

where we have used the expansion of $\gamma(\tau)$ and the condition $f_{p_{1}}^{-1}\left(\tau_{c}\right) \simeq 0$. We can check numerically that this is the correct behavior. In order to do so, we first fit the calculated function $f_{p_{1}}^{-1}(\tau)$ near $\tau_{c}$ with a linear form, $\gamma_{c}\left(\tau_{c}-\tau\right)$, and get the onset time $\tau_{c} \simeq 0.1006$ as well as $\gamma_{c} \simeq-0.6$. Then we compare these values to those obtained from the fit of the quantity $3\left(I_{a}-I_{b} T^{*}\right) /\left(p_{1} T^{*}\right)$ which exhibits a linear time dependence near $\tau_{c}$, as expected. The two values agree perfectly, as can be seen in Fig A.8.

It is interesting to compare the present analysis to the analogous one presented in Ref. [2]. In this case, $p_{1} / m \rightarrow 1$, and the combination $I_{a}-T^{*} I_{b}$ vanishes. Both in the massive and the massless case, one finds that $\mu^{*}-m$ vanishes linearly with $\tau_{c}-\tau$, but while this result follows from a simple argument in the massive case, in the massless case this could only be determined numerically. 


\section{Appendix B. Appendix B: Details of Numerics}

In this Appendix, we give some details on how we solve the Fokker-Planck equation. An efficient strategy is to solve its "integrated version". Namely, instead of solving the differential equation for the distribution function directly, we evaluate the total number of particles in a thin shell $\int_{p-\Delta p / 2}^{p+\Delta p / 2} d p p^{2} f(p) \approx$ $p^{2} \Delta p f(p)$ and examine its time evolution by integrating the transport equation over this moment window:

$$
p^{2} \Delta p \partial_{\tau} f(p)=\left(p^{2} \mathcal{J}\right)_{p-\Delta p / 2}^{p+\Delta p / 2}=\mathcal{F}(p+\Delta p / 2)-\mathcal{F}(p-\Delta p / 2)
$$

Note on the right-hand side the kernel (taking the form of a full derivative $\sim \nabla \cdot \mathcal{J}$ ) will be integrated to give the difference of the flux $\mathcal{F}=p^{2} \mathcal{J}$ on the two surfaces of this shell at $p \pm \Delta p / 2$ respectively.

Numerically we discretize the distribution on an equally spaced momentum grid $p_{i}=(i-1 / 2) \Delta p, i=1, \ldots, 400$, where $\Delta p=0.01$. So the flux is on the grid $p_{i}=i \Delta p, i=0,1, \ldots, 400$. It is easy to see that $\mathcal{F}(p=0)=0$. We further set the flux to vanish at our momentum grid's UV cutoff, i.e. $\mathcal{F}\left(p=\Lambda=4 Q_{s}\right)=0$, as the boundary condition that enforces exact particle number conservation. This UV cutoff scale is also used for the regulating the $q$-integration in the collision kernel. Note that the $q$-integration gives essentially the familiar Coulomb logarithm, and the cutoff effect scales as $\sim \log (\Lambda / m)$. Varying the cutoff would only bring rather minor change in the overall evolution time scale in a logarithmic way, and will lead to no other change in the evolution dynamics (in particular no change to the scaling behavior when approaching onset), as indeed demonstrated by our test computations.

To solve this equation we use the implicit Gaussian scheme (which is a standard algorithm for this type of equation) as

$$
f_{\tau+\delta \tau}\left(p_{i}\right)-f_{\tau}\left(p_{i}\right)=\frac{\delta \tau}{p^{2} \Delta p}\left(\mathcal{F}_{p_{i}+\Delta p / 2}\left[f_{\tau+\delta \tau}\right]-\mathcal{F}_{p_{i}-\Delta p / 2}\left[f_{\tau+\delta \tau}\right]\right)
$$

where $\mathcal{F}_{p}\left[f_{\tau+\delta \tau}\right]$ is the flux at momentum $p$ evaluated with the distribution at $\tau+\delta \tau$. The implicit scheme then involves numerically solving the above set of equations to extract the distribution at time $\tau+\delta \tau$. The advantage of this implicit scheme, as is well know, is its robust numerical stability as compared with e.g. the explicit scheme of directly evolving the equation in time. During the whole evolution we have implemented an automated adjustment of the time step $\delta \tau$ to guarantee that the distribution function at the lowest grid point (which has the largest occupation $f(p)) p=\Delta p / 2$ varies less than $5 \%$ at each time step forward. This allows rather accurate handling of the very infrared part of the evolution which is important for understanding the critical behavior in the onset of condensation. In the entire calculation the particle number conservation is exact while the energy conservation is maintained at the order of $10^{-3}$ variation or less. Furthermore this method is relatively insensitive to choice of the momentum grid, as we have tested by changing the momentum grid into a finer one. 


\section{References}

[1] J. -P. Blaizot, F. Gelis, J. -F. Liao, L. McLerran, and R. Venugopalan, Nucl. Phys. A 873, 68 (2012). [arXiv:1107.5296 [hep-ph]].

[2] J. -P. Blaizot, J. Liao, and L. McLerran, Nucl. Phys. A 920, 58 (2013) [arXiv:1305.2119 [hep-ph]].

[3] A. H. Mueller, A. I. Shoshi, and S. M. H. Wong, Phys. Lett. B632, 257-260 (2006). [hep-ph/0505164]; A. H. Mueller, A. I. Shoshi, and S. M. H. Wong, Eur. Phys. J. A29, 49-52 (2006). [hep-ph/0512045]. A. H. Mueller, A. I. Shoshi, and S. M. H. Wong, Nucl. Phys. B760, 145-165 (2007). [hep-ph/0607136].

[4] J. P. Blaizot, B. Wu and L. Yan, Nucl. Phys. A 930, 139 (2014) [arXiv:1402.5049 [hep-ph]].

[5] F. Scardina, D. Perricone, S. Plumari, M. Ruggieri and V. Greco, Phys. Rev. C 90, no. 5, 054904 (2014) [arXiv:1408.1313 [nucl-th]].

[6] X. G. Huang and J. Liao, arXiv:1303.7214 [nucl-th].

[7] Z. Xu, K. Zhou, P. Zhuang and C. Greiner, arXiv:1410.5616 [hep-ph].

[8] A. Kurkela and G. D. Moore, Phys. Rev. D 86, 056008 (2012) [arXiv:1207.1663 [hep-ph]].

[9] M. C. A. York, A. Kurkela, E. Lu and G. D. Moore, Phys. Rev. D 89, 074036 (2014) [arXiv:1401.3751 [hep-ph]].

[10] J. Berges, J. P. Blaizot and F. Gelis, J. Phys. G 39, 085115 (2012) [arXiv:1203.2042 [hep-ph]].

[11] X. G. Huang and J. Liao, Int. J. Mod. Phys. E 23, 1430003 (2014) [arXiv:1402.5578 [nucl-th]].

[12] D. V. Semikoz and I. I. Tkachev, Phys. Rev. Lett. 74, 3093 (1995); Phys. Rev. D 55, 489 (1997).

[13] C. W. Gardiner, M. D. Lee, R. J. Ballagh, M. J. Davis and P. Zoller, Phys. Rev. Lett. 81, 5266 (1998); C. W. Gardiner, P. Zoller, R. J. Ballagh and M. J. Davis, Phys. Rev. Lett. 79, 1793 (1997).

[14] J. Berges, K. Boguslavski, S. Schlichting, and R. Venugopalan, arXiv:1303.5650 [hep-ph].

[15] T. Epelbaum and F. Gelis, Phys. Rev. Lett. 111, 232301 (2013) [arXiv:1307.2214 [hep-ph], arXiv:1307.2214 [hep-ph]].

[16] T. Gasenzer, L. McLerran, J. M. Pawlowski and D. Sexty, Nucl. Phys. A (2014) [arXiv:1307.5301 [hep-ph]]. 
[17] J. -P. Blaizot, J. Liao, and L. McLerran, in preparation.

[18] A. H. Mueller, Nucl. Phys. B 572, 227 (2000) [arXiv:hep-ph/9906322]; Phys. Lett. B 475, 220 (2000) [arXiv:hep-ph/9909388].

[19] E.M. Lifshitz and L.P. Pitaevskii, Physical Kinetics (Pergamon Press, New York, 1981). 